\title{
Three-dimensional $\mathrm{MgB}_{2}$-type superconductivity in hole-doped diamond.
}

\author{
Lilia Boeri, ${ }^{1,2}$ Jens Kortus,, , 6 and O. K. Andersen ${ }^{2}$ \\ ${ }^{I}$ INFM Center for Statistical Mechanics and Complexity and Dipartimento di Fisica, \\ Università di Roma "La Sapienza", Piazzale A. Moro 2, 00185 Roma, Italy \\ ${ }^{2}$ Max-Planck Institut für Festkörperforschung, Heisenbergstr. 1, D-70569 Stuttgart, Germany
}

(Dated: October 29, 2018)

\begin{abstract}
We substantiate by numerical and analytical calculations that the recently discovered superconductivity below $4 \mathrm{~K}$ in $3 \%$ boron-doped diamond is caused by electron-phonon coupling of the same type as in $\mathrm{MgB}_{2}$, albeit in 3 dimensions. Holes at the top of the zone-centered, degenerate $\sigma$-bonding valence band couple strongly to the optical bond-stretching modes. The increase from 2 to 3 dimensions reduces the mode-softening crucial for $T_{c}$ reaching $40 \mathrm{~K}$ in $\mathrm{MgB}_{2}$. Even if diamond had the same bare coupling constant as $\mathrm{MgB}_{2}$, which could be achieved with $10 \%$ doping, $T_{c}$ would only be $25 \mathrm{~K}$. Superconductivity above $1 \mathrm{~K}$ in $\mathrm{Si}(\mathrm{Ge})$ requires hole-doping beyond $5 \%(10 \%)$.
\end{abstract}

PACS numbers: 74.70.-b,74.70.Ad,74.25.Kc

Recently, superconductivity below $T_{c} \sim 4 \mathrm{~K}$ was reported in diamond doped with $x \sim 3 \%$ boron, that is, with $\sim 0.03$ holes per carbon atom [1]. Such high hole-doping levels can be achieved due to the small size of boron. It had previously been observed that the prominent Raman line caused by the zonecenter optical phonons at $1332 \mathrm{~cm}^{-1}$ downshifts and broadens significantly upon heavy boron doping [2]. In this Letter we shall make plausible that the superconductivity in hole-doped diamond is due to the coupling of the holes to the optical zonecenter phonons, a mechanism similar to the one causing hightemperature superconductivity in $\mathrm{MgB}_{2}$, but without some of its interesting features. We shall also estimate transition temperatures for hole-doped $\mathrm{Si}$ and $\mathrm{Ge}$.

The discovery [3] of superconductivity below $40 \mathrm{~K}$ in $\mathrm{MgB}_{2}$, a binary compound isostructural and isoelectronic with graphite, came as a surprise for the scientific community. By now, it is well understood what the mechanism is and why $\mathrm{MgB}_{2}$ is special [4, 5, 6, 7, 8]: In contrast to other known $s p^{2}$-bonded superconductors, such as intercalated graphite, alkali doped fullerides, and organic superconductors whose charge carriers are exclusively $\pi$-electrons, $\mathrm{MgB}_{2}$ has holes at the top of the bonding $\sigma$-bands at the zone center. These holes, on two narrow Fermi cylinders with radii $\sim 1 / 5$ of the Brillouin-zone radius, couple strongly $(\lambda \sim 1)$ to the two optical bond-stretching modes with $q \leq 2 k_{F} \ll k_{B Z}$, giving rise to a strong 2-dimensional Kohn anomaly in the phonon spectrum. This strong coupling between a few zone-center holes and optical phonons is what drives the high-temperature superconductivity in $\mathrm{MgB}_{2}$. Experience shows [5], and it can be proved for parabolic bands with $2 k_{F} \ll k_{B Z}$ [9], that the coupling constant is given by the Hopfield expression,

$$
\lambda=\frac{N D^{2}}{M \omega^{2}},
$$

where $N$ is the density of states (DOS) per spin at the Fermi level of the $\sigma$ holes. Moreover, $\pm D u$ is the splitting of the degenerate top of the $\sigma$-band by the displacement $\mathrm{e} u$ of a frozen, optical zone-center phonon with normalized eigenvector e and energy $\omega$. The optical phonons are softened by the interac- tion with the holes, $\omega^{2} \sim \omega_{0}^{2} /(1+2 \lambda)$ when $q<2 k_{F}$, and that significantly enhances $\lambda$ and $T_{c} \sim \omega \exp (-1 / \lambda)$. This softening is presumably weakened by anharmonicity [10, 11]. The DOS is independent of doping because the $\sigma$-band is 2 dimensional. As a consequence, a decrease in the number of holes, e.g. by carbon-doping, should not cause $\lambda$ to decrease, except through the anharmonic hardening of $\omega$ caused by the decrease of $E_{F}$ [12]. In stoichiometric $\mathrm{MgB}_{2}$ there are more carriers in the $\pi$ bands than in the $\sigma$ bands $(0.09$ per B), but the former couple far less to phonons than the latter, and since there seems to be very little impurity scattering between the $\sigma$ and $\pi$-bands, $\mathrm{MgB}_{2}$ is the first superconductor which clearly exhibits multiple gaps below a common $T_{c}$ 17, 8, 10, 13].

Instead of having $\pi$-bands and three 2-dimensional bonding $\sigma$-bands, $s p^{3}$-bonded semiconductors like diamond have four 3-dimensional bonding $\sigma$-bands. The top of this valence band is three-fold degenerate with symmetry $T_{2 g}$, and so are the zone-center optical phonon modes. Like in $\mathrm{MgB}_{2}, \sigma$-holes with small $k_{F}$ should therefore couple strongly, and for small $k_{F}$ exclusively, to the optical bond-stretching modes, with the main differences being that in 3 dimensions the Kohn anomaly is weaker and the DOS increases with hole doping like $k_{F}$, the radius of the average Fermi sphere. Since there are 3 bands and two carbon atoms per cell, $\left(k_{F} / k_{B Z}\right)^{3}=x / 3$. For $x=0.03, k_{F} / k_{B Z}$ is 0.22 , which is like in $\mathrm{MgB}_{2}$. Due to the lack of a metallic $\pi$-band, diamond becomes an insulator once $x$ is below 1-2 per cent. We shall now substantiate this scenario for the observed superconductivity in hole-doped diamond by providing quantitative details, and we shall also consider the possibility of superconductivity in hole-doped $\mathrm{Si}$ and Ge. In particular, we shall present results of density-functional (LDA) calculations and estimate $T_{c}$ using Eliashberg theory.

A substitutional boron impurity in diamond has an acceptor level with binding energy $0.37 \mathrm{eV}$ [14]. With increased doping, the boron impurity band will eventually overlap the diamond valence band and the system becomes metallic at a boron concentration of $8 \cdot 10^{20} \mathrm{~cm}^{-3}$. Since this is one order of magnitude lower than the doping at which superconductivity was observed [1], we felt justified in using a virtual crystal 
approximation (VCA) in which the carbon nuclei have charge $6-x$ and the crystal is neutral.

The valence bands were calculated with the scalarrelativistic full-potential LMTO method [15], and the phonon dispersions and the electron-phonon spectral function $\alpha^{2} F$ were calculated with the linear-response method [15]. Effects of anharmonicity were considered in a second step. We used a triple- $\kappa$ sp $d$ LMTO basis set and represented the charge densities and potentials by spherical harmonics with $l \leq 6$ inside non-overlapping muffin-tin spheres and by plane waves with energies less than 400 Ry between the spheres. The resulting band structure for undoped diamond agrees with those of earlier LDA calculations. Due to the smallness of $k_{F}$, we needed to use a fine $\mathbf{k}$-mesh chosen as a $1 / 32^{3}$ sublattice in reciprocal space. $\alpha^{2} F$ is evaluated as a weighted sum over linewidths of individual phonons, and for this a fine, yet affordable q-mesh is needed. It was chosen as a $1 / 8^{3}$ sublattice in reciprocal space. The $\lambda$-values obtained herewith are accurate when $x \gtrsim 0.05$, whereas Eq. (1) which we have now derived analytically also in three dimensions, is more accurate for smaller dopings. Since, even for 10\% doping, we calculated an increase of the lattice constant by less than a per cent, we did use the experimental lattice parameters for the undoped materials in all subsequent calculations .

In Fig. 11 we show the top of the valence-band structure calculated for $10 \%$ hole-doped diamond. For this unrealistically heavy doping, $N$ reaches $75 \%$ of the $\sigma$-band DOS in $\mathrm{MgB}_{2}$. The electronic parameters may be found together with those for $\mathrm{MgB}_{2}$ in the first columns of the table. Due to the deviations from parabolicity seen in Fig. 1 $N$ decreases somewhat faster than $x^{1 / 3}$. As is well known, the LDA gap is too small and this leads to a slight underestimate of the valence-band masses and the DOS. Nevertheless, properties derived from the total energy, like phonon energies, are quite accurate.

For the displacement $\mathbf{e} u$ of a frozen, optical zone-center phonon with two bonds stretched and two contracted, say those in respectively the positive and negative $z$-directions, the top of the valence band is deformed as shown in Fig. 11 For small $k$ there are two identical bands, split in energy by

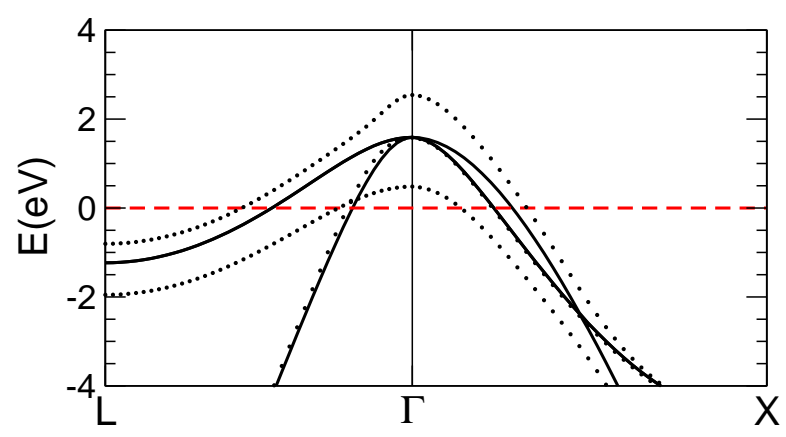

FIG. 1: LDA band-structure of diamond with $x=0.1$ holes/C (full lines). A frozen optical zone-center phonon with two bonds stretched and two contracted by $u / \sqrt{2}$ splits the bands by $\pm D u$ (dotted lines). $u=0.05 \AA$. $\pm D u$, and a band which does not move with respect to the Fermi level. At $\Gamma$ the corresponding wavefunctions are those linear combinations of the bond orbitals which have respectively $\left(p_{x} \pm p_{y}\right) \sqrt{2}$ and $p_{z}$ symmetry. The value of $D$ given in the table is seen to be nearly twice as large as in $\mathrm{MgB}_{2}$. For pure diamond it agrees with the accepted value [16], and it is seen to decrease slightly with doping.

In Fig. 2 we show the phonon dispersions calculated in the harmonic approximation for undoped and hole-doped diamond. The dispersions for pure diamond, including the slight upturn of the uppermost mode when moving away from the zone center [17], are well reproduced, and for the frequency $1332 \mathrm{~cm}^{-1}$ of the optical zone-center modes we calculate $\omega_{0}=1292 \mathrm{~cm}^{-1}$. Previous LDA calculations [18] obtained similar results. In the presence of hole-doping, the calculated dispersions of the optical modes clearly exhibit softening near the zone center and a 3-dimensional Kohn anomaly around $q=2 k_{F}$.

The softening of the zone-center phonons is $(2 / 3) \lambda$ instead of $\lambda$, as in the case of $\mathrm{MgB}_{2}$. This is most easily seen by considering a frozen phonon calculation and Fig. 11 The adiabatic redistribution of $(1 / 3) 2 N D u$ electrons from the upper third to the lower third of the deformed valence band decreases the energy of each electron by $D u$, and therefore perturbs the potential energy of the harmonic oscillator by $-(1 / 3) 2 N D^{2} u^{2}$. As a consequence, $(1 / 2) M \omega^{2}=(1 / 2) M \omega_{0}^{2}-(1 / 3) 2 N D^{2}$, and by use of Eq. (1) we get: $\omega^{2}=\omega_{0}^{2} /\left(1+2 \frac{2}{3} \lambda\right)$. In $\mathrm{MgB}_{2}$ no part of the $\sigma$-band is passive in the screening of the phonon, so the factor $2 / 3$ is missing. The value of $\lambda$ deduced from the frequencies, $\omega$ and $\omega_{0} \equiv \omega(x=0)$, of the optical zone-center modes calculated by linear response is given in the table $\left(\lambda_{\omega}\right)$. This $\lambda_{\omega}$-value is seen to agree well with the value $\lambda_{D}$ obtained by use of Eq. (1). In order to separate the materials and dimensional dependencies of $\lambda$, we express it in terms of a bare coupling constant, $\lambda_{0}$, and an enhancement due to the phonon softening:

$$
\lambda_{0} \equiv \frac{N D^{2}}{M \omega_{0}^{2}}, \quad \lambda=\frac{\lambda_{0}}{1-2 \frac{2}{3} \lambda_{0}}, \quad \frac{\omega^{2}}{\omega_{0}^{2}}=1-2 \frac{2}{3} \lambda_{0} .
$$

The enhancement is weaker in 3 dimensions than in 2, where the reduction factor $2 / 3$ is missing. As for the materials dependence, the $\lambda_{0}$-values given in the table first of all show that $10 \%$ doped diamond has the same $\lambda_{0} \sim 1 / 3$ as $\mathrm{MgB}_{2}$ : The bare force constant, $M \omega_{0}^{2}$, is 0.49 times its value in $\mathrm{MgB}_{2}, N$ is 0.75 , and $D$ is 1.65 . Due to the difference in dimensionality, $\lambda \sim \frac{1 / 3}{1-4 / 9}=0.6$ in doped diamond, but $\lambda \sim \frac{1 / 3}{1-2 / 3}=1$ in $\mathrm{MgB}_{2}$. With decreasing doping in diamond, $N$ decreases roughly like $x^{1 / 3}, D$ increases slightly, and $M \omega_{0}^{2}$ is constant. As a consequence, for $3 \%$ doping $\lambda_{0}$ is only 0.21 and $\lambda$ is 0.30 .

We can also calculate the electron-phonon spectral function and $\lambda=2 \int \omega^{-1} \alpha^{2} F(\omega) d \omega$ numerically by sampling over all phonon branches and energy bands. The result shown in Fig. 3 confirms that only the optical phonons interact with the holes: $\alpha^{2} F$ vanishes for phonon frequencies below that 
TABLE I: $N$ is in states/eV/spin/f.u. $D$ is in eV/A. $\omega$ is in $\mathrm{cm}^{-1} . \lambda_{0}$ is the bare electron-phonon coupling constant defined in Eq. (2). $\lambda_{D}$ and $\lambda_{\omega}$ are estimates of the coupling constant as obtained from respectively Eq. 11 and the softening of $\omega$. $\lambda$ is obtained from the numerical linearresponse calculation and includes all phonons and $\sigma$-electrons; for $\operatorname{MgB}_{2}$ it is $\lambda_{\sigma \sigma}[21] . a \equiv E_{F}^{\prime} / D t$. $\lambda_{a}$ is $\lambda$ corrected for anharmonicity using Eq. 3. $T_{c}$ is obtained from Eq. 4 using $\lambda_{a}, \omega_{a}$, and $\mu^{*}=0.1$.

\begin{tabular}{rcccccccccc}
\hline \hline & $N$ & $D$ & $\omega$ & $\lambda_{0}$ & $\lambda_{D}$ & $\lambda_{\omega}$ & $\lambda$ & $a$ & $\lambda_{a}$ & $T_{c}$ \\
$\mathrm{MgB}_{2}$ & 0.15 & 12.4 & 536 & 0.33 & 1.01 & - & 1.02 & 0.9 & 0.78 & 45 \\
$\mathrm{C}$ & 0.00 & 21.6 & 1292 & 0 & 0 & 0 & 0 & 0.0 & 0 & 0 \\
$3 \% \mathrm{C}$ & 0.07 & 21.1 & 1077 & 0.21 & 0.30 & 0.33 & 0.30 & 0.7 & 0.27 & 0.2 \\
$5 \% \mathrm{C}$ & 0.08 & 20.8 & 1027 & 0.25 & 0.37 & 0.44 & 0.36 & 0.9 & 0.33 & 2 \\
$10 \% \mathrm{C}$ & 0.11 & 20.4 & 957 & 0.32 & 0.57 & 0.62 & 0.56 & 1.3 & 0.54 & 25 \\
$\mathrm{Si}$ & 0.00 & 6.8 & 510 & 0 & 0 & 0 & 0 & 0.0 & 0 & 0 \\
$5 \% \mathrm{Si}$ & 0.17 & 6.3 & 453 & 0.13 & 0.16 & 0.20 & 0.30 & 1.4 & 0.30 \\
$10 \% \mathrm{Si}$ & 0.24 & 6.1 & 438 & 0.17 & 0.22 & 0.27 & 0.40 & 2.0 & 0.40 \\
$\mathrm{Ge}$ & 0.00 & 5.8 & 317 & 0 & 0 & 0 & 0 & 0.0 & 0 \\
$10 \% \mathrm{Ge}$ & 0.20 & 4.4 & 282 & 0.08 & 0.09 & 0.20 & 0.32 & 5.1 & 0.32 \\
\hline \hline
\end{tabular}

of the optical zone-center modes, then jumps to a maximum, and finally falls. The decay occurs more slowly than in $\mathrm{MgB}_{2}$ due to the increase of dimensionality. The $\lambda$-values $(\lambda)$ obtained from this calculation again agree well with those obtained from Eq. (1) and from the phonon softening.

In $\mathrm{MgB}_{2}$ the role of anharmonicity of the optical phonon modes with $q<2 k_{F}$ has been stressed [10]; it hardens the phonon by about $20 \%$ and thus decreases $\lambda$ from 1.0 to $\lambda_{a}=0.78$, as given in the table. However, this has recently been questioned [11]. While anharmonicity may be crucial in $\mathrm{MgB}_{2}$ it has at most a noticeable effect on the superconductivity in diamond at small dopings $(x<3 \%)$ as we shall now see: The anharmonicity appears in frozen phonon calculations (see Fig. 1), because once the displacement $u$ exceeds $E_{F} / D$, the lower band is full so that the screening is lost [12]. In the expression for the perturbation of the potential energy of the oscillator, $u^{2}$ should now be substituted by

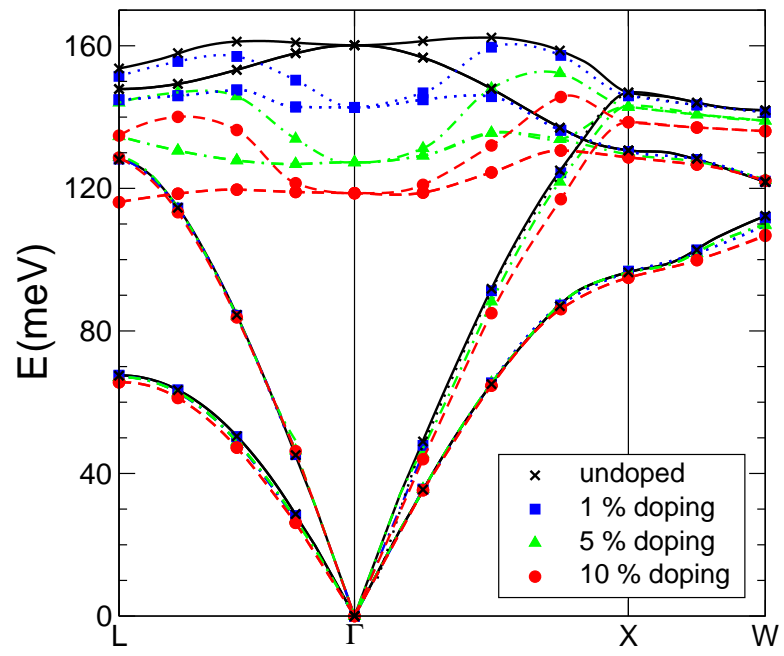

FIG. 2: Phonon dispersions calculated with the linear-response method for diamond with $x=0,1,5$, and $10 \%$ hole doping.
$\left(|u|-E_{F} / D\right)^{2} \theta\left(E_{F} / D-|u|\right)$, provided that we simplify the DOS shape by a square. It has been shown that the most important anharmonic contribution to $T_{c}$ is the decrease of the first excitation energy [19]. By first-order perturbation theory, this is simply $(1 / 3) 2 N D^{2} t^{2}\left[1-\operatorname{erf}\left(E_{F} / D t\right)\right]$, where $t \equiv \sqrt{\hbar / M \omega}$ is the classical turning point in the ground state. Introducing again Eq. (1) we obtain the result:

$$
\frac{\lambda_{a}}{\lambda}=\frac{\omega^{2}}{\omega_{a}^{2}}=\frac{1}{1+2(2 / 3) \lambda\left[1-\operatorname{erf}\left(E_{F} / D t\right)\right]} .
$$

For $\mathrm{MgB}_{2}$ the assumption of a square $N(E)$ is good, but due to the missing factor $2 / 3$ and the presence of the $\pi$-band, $(2 / 3)$ in Eq. (3) should be substituted by $\left[1-N_{\pi} /\left(N+2 N_{\pi}\right)\right]$. For hole-doped diamond, $N(E)$ has square-root shape, and this we crudely take into account by substituting $E_{F}$ in Eq. (3) by $E_{F}^{\prime}=(2 / 3) E_{F}$. In the table we have included the ratio $E_{F}^{\prime} / D t \equiv a$ as well as the results for $\lambda_{a}$. We see that the effect of anharmonicity may be important in $\mathrm{MgB}_{2}$ but merely noticeable in hole-doped diamond [20].

For this type of superconductivity, which is characterized by an Eliashberg function with the shape exhibited in Fig. 3 and which we can idealize by a $\delta$-function at the frequency $\omega$ of the optical zone-boundary phonon, solution of the Eliashberg equations yields with high accuracy:

$$
T_{c}=\omega \exp \left(\frac{-1}{\frac{\lambda}{1+\lambda}-\mu^{*}}\right) .
$$

This is McMillan's expression with all numerical factors, which he obtained by fitting to $F(\omega)$ of niobium, set equal to unity. For the cases considered in the present paper, it does not make much difference whether one uses McMillan's factors or unity inside the exponential, but it is important that the prefactor is $\omega$, rather than $\left\langle\omega_{\ln }\right\rangle / 1.2$.

We can finally estimate $T_{c}$ from Eq. (4) with the values for $\lambda_{a}$ given in the table and $\omega_{a}$ from Eq. (3). For the Coulomb pseudopotential, the standard value $\mu^{*}=0.1$ was used in all cases. For $\mathrm{MgB}_{2}$ we neglected the $\pi$-bands. Considering the 


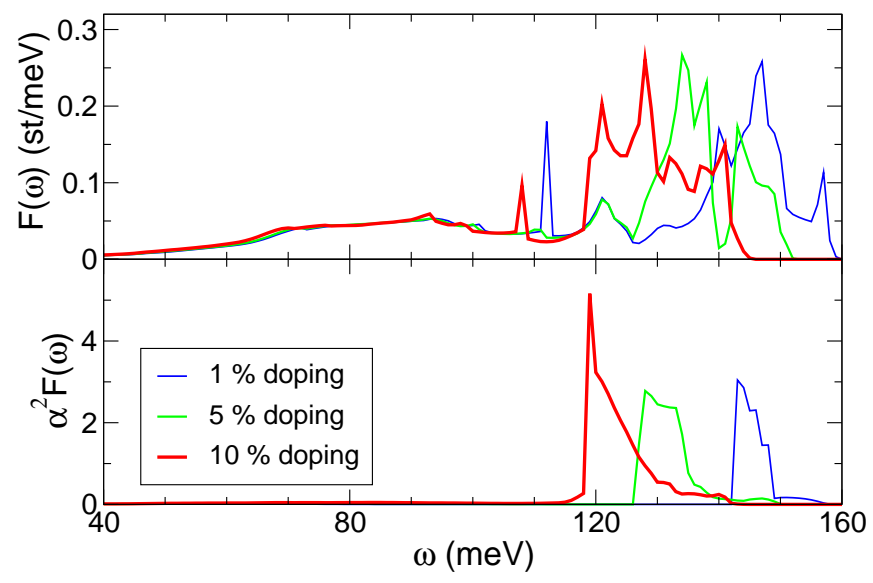

FIG. 3: The phonon density of states, $F(\omega)$, and Eliashberg function, $\alpha^{2} F(\omega)$, calculated numerically by linear response considering all electrons and phonons.

uncertainties in our calculation of $\lambda$ and $\omega$, the uncertainty of $\mu^{*}$, and the experimental estimation of the doping level, we do find critical temperatures in good agreement with present experimental knowledge. We therefore believe to have substantiated our claim that the superconductivity in hole-doped diamond is of $\mathrm{MgB}_{2}$-type, but in three dimensions.

We repeated our calculations for hole-doped $\mathrm{Si}$ and $\mathrm{Ge}$, and include those results in the table for which $E_{F}$ largely exceeds the spin-orbit splitting, which we neglected. Whereas hole-doped $\mathrm{C}$ shows superconductivity above $1 \mathrm{~K}$ for doping levels presently obtainable, $\mathrm{Si}$ and $\mathrm{Ge}$ seem to need twice as high doping levels. The main reason is that the deformation potential in $\mathrm{Si}$ and $\mathrm{Ge}$ is about four times smaller than in $\mathrm{C}$, which is too small to take advantage of having twice as large a DOS and a three times smaller force-constant. There is also a qualitative difference to diamond: For heavily doped Si and Ge, the holes not only couple to the optical, but also to the acoustic phonons. This is the reason why $\lambda$ exceeds $\lambda_{D} \sim \lambda_{\omega}$.

In conclusion, we have shown that the recently discovered superconductivity in hole-doped diamond below $4 \mathrm{~K}$ is of $\mathrm{MgB}_{2}$-type, but in three dimensions. This means that the mechanism is coupling of a few holes at top of the $\sigma$ bonding valence band to the optical bond-stretching zonecenter phonons. The increase from 2 to 3 dimensions limits the strong softening of the optical modes mainly responsible for the high $T_{c}$ in $\mathrm{MgB}_{2}$. On the other hand, the deformation potentials in diamond are twice stronger than in $\mathrm{MgB}_{2}$. Kelvin-range superconductivity in $\mathrm{Si}$ and $\mathrm{Ge}$ would require hole-doping levels of 5-10\%. Finally, we have obtained sim- ple analytical expressions for $\mathrm{MgB}_{2}$-type superconductivity.

A purely electronic mechanism for the observed superconductivity was recently suggested [22], and after submission of the present manuscript two works similar to ours appeared [23, 24]. The latter used a supercell approach to simulate the boron doping and found an electron-phonon coupling in very good agreement with our results.

We are grateful to O. Dolgov, M. Cardona, G. B. Bachelet, E. Cappelluti, and L. Pietronero for many interesting discussions.

* Electronic address: j.kortus@ @ fkf.mpg.de

[1] E. A. Ekimov et al., Nature (London), 428, 542 (2004).

[2] R. J. Zhang, S. T. Lee, and Y. W. Lam, Diamond Rel. Mater. 5, 1288 (1996).

[3] J. Nagamatsu et al., Nature (London) 410, 63 (2001).

[4] J. Kortus et al., Phys. Rev. Lett. 86, 4656 (2001).

[5] J. M. An, W. E. Pickett, Phys. Rev. Lett. 86, 4366 (2001).

[6] Y. Kong et al., Phys. Rev. B 64, 020501 (R) (2001).

[7] A. Y. Liu, I. I. Mazin and J. Kortus, Phys. Rev. Lett. 87, 087005 (2001).

[8] P. C. Canfield and G. W. Crabtree, Physics Today 56 March, 34 (2003).

[9] O. K. Andersen et al. (unpublished)

[10] H.J. Choi et al., Phys. Rev. B 66, 020513 (2002); H.J. Choi et al., Nature (London) 418, 758 (2002).

[11] M. Lazzeri, M. Calandra, and F. Mauri, Phys. Rev. B 68, 220509 (2003).

[12] L. Boeri et al., Phys. Rev. B 65, 214501 (2002).

[13] I.I. Mazin et al., Phys. Rev. Lett. 89, 107002 (2002).

[14] F. Fontaine, J. Appl. Phys. 85, 1409 (1999).

[15] S. Y. Savrasov, Phys. Rev. B 54, 16470 (1996).

[16] M. Cardona and N. E. Christensen, Solid State Commun. 58, 421 (1986); O. H. Nielsen and R. M. Martin, Phys. Rev. B 32, 3792 (1985); F. Cerdeira and M. Cardona, Phys. Rev. B 5, 1440 (1972).

[17] M. Schwoerer-Bohning, A. T. Macrander, and D. A. Arms; Phys. Rev. Lett. 80, 5572 (1998).

[18] P. Pavone et al., Phys. Rev. B 48, 3156 (1993); W. Windl et al., Phys. Rev. B 48, 3164 (1993); G. Kresse, J. Furthmüller, and J. Hafner, Europhys. Lett. 32, 729 (1995).

[19] J. C. Hui and P. B. Allen, J. Phys. F: Metal Phys., 4, L42 (1974).

[20] The anharmonicity mechanism [12] is different from the one discussed for pure semiconductors, in e.g. G. Lang et al., Phys. Rev. B 59, 6182 (1999).

[21] A.A. Golubov et al., J. Phys.: Condens. Matter 14, 1353 (2002).

[22] G. Baskaran, cond-mat/0404286.

[23] K.-W. Lee, W. E. Pickett, cond-mat/0404547.

[24] H. J. Xiang et al., cond-mat/0406446 